Elect. Comm. in Probab. 16 (2011), 741-752

ELECTRONIC

COMMUNICATIONS

in PROBABILITY

\title{
HARD EDGE TAIL ASYMPTOTICS
}

\author{
JOSÉ A. RAMÍREZ
}

Department of Mathematics, Universidad de Costa Rica

email: alexander.ramirez_g@ucr.ac.cr

BRIAN RIDER $^{1}$

Department of Mathematics, University of Colorado Boulder

email: brian.rider@colorado.edu

OFER ZEITOUNI ${ }^{2}$

Department of Mathematics, University of Minnesota \& Weizmann Institute

email: zeitouni@math.umn.edu

Submitted September 19, 2011, accepted in final form November 4, 2011

AMS 2000 Subject classification: 60B20, 60F10

Keywords: Random matrices, smallest singular value, hard edge

\section{Abstract}

Let $\Lambda$ be the limiting smallest eigenvalue in the general $(\beta, a)$-Laguerre ensemble of random matrix theory. That is, $\Lambda$ is the $n \uparrow \infty$ distributional limit of the (scaled) minimal point drawn from the density proportional to $\prod_{1 \leq i<j \leq n}\left|\lambda_{i}-\lambda_{j}\right|^{\beta} \prod_{i=1}^{n} \lambda_{i}^{\frac{\beta}{2}(a+1)-1} e^{-\frac{\beta}{2} \lambda_{i}}$ on $\left(\mathbb{R}_{+}\right)^{n}$. Here $\beta>0, a>-1$; for $\beta=1,2,4$ and integer $a$, this object governs the singular values of certain rank $n$ Gaussian matrices. We prove that

$$
P(\Lambda>\lambda)=e^{-\frac{\beta}{2} \lambda+2 \gamma \sqrt{\lambda}} \lambda^{-\frac{\gamma(\gamma+1-\beta / 2)}{2 \beta}} \mathfrak{e}(\beta, a)(1+o(1))
$$

as $\lambda \uparrow \infty$ in which $\gamma=\frac{\beta}{2}(a+1)-1$ and $\mathfrak{e}(\beta, a)>0$ is a constant (which we do not determine). This estimate complements/extends various results previously available for special values of $\beta$ and $a$.

\section{Introduction}

The shape of the distribution of the smallest singular value of a "typical" matrix is a deeply studied question. An overview of the varying motivations for this problem may be found in [11]. In the case of Gaussian matrices, many exact formulas are available both at finite dimension and asymptotically $[4,13]$. Only quite recently has it been shown that the asymptotic laws are universal beyond the Gaussian case (in the sense of being insensitive to the statistics of the matrix entries), see [12].

\footnotetext{
${ }^{1}$ RESEARCH SUPPORTED IN PART BY NSF GRANT DMS-0645756.

${ }^{2}$ RESEARCH SUPPORTED IN PART BY NSF GRANT DMS-0804133.
} 
Here we consider the "general beta" analogues of the classical Gaussian ensembles. These are defined by placing a measure on $n$ nonnegative real points $\lambda_{1}, \lambda_{2}, \ldots, \lambda_{n}$ with density function (a normalization constant times)

$$
\prod_{1 \leq i<j \leq n}\left|\lambda_{i}-\lambda_{j}\right|^{\beta} \prod_{i=1}^{n} \lambda_{i}^{\frac{\beta}{2}(a+1)-1} e^{-\frac{\beta}{2} \lambda_{i}} .
$$

When $\beta=1,2,4$ and $a=0,1,2, \ldots$ this is the joint square-singular value law of an $n \times(n+a)$ real, complex, or quaternion Gaussian matrix. It is however a sensible law for any $\beta>0$ and $a>-1$, and, what is more, still a joint square-singular value law for a certain random bi-diagonal matrix ensemble [3]. Further, the least order statistic $\lambda_{\min }$ satisfies a limit law: as $n \uparrow \infty, n^{2} \lambda_{\text {min }}$ converges (in distribution) to a well defined random variable, denoted here by $\Lambda(=\Lambda(\beta, a))$. There are several proofs of this for special values of $\beta$ and $a$; [10] contains a proof (making use of the bi-diagonal representation of [3] and substantiating a conjecture of [5]) valid for all values of those parameters.

Our starting point is a relation between the law of $\Lambda$ and the explosion/non-explosion of the diffusion process: with $b$ a Brownian motion,

$$
d x(t)=d b(t)+\left(\frac{\beta}{4}\left(a+\frac{1}{2}\right)-\frac{\beta}{2} \sqrt{\lambda} e^{-\beta t / 8} \cosh x(t)\right) d t .
$$

In particular, a corrected version of Theorem 2 of [10] (see also the derivation leading to (7) below) implies that

$$
P(\Lambda>\lambda)=\mathbb{P}_{\infty, 0}(t \mapsto x(t) \text { never hits }-\infty) .
$$

Here $\mathbb{P}_{c, s}$ indicates the law on paths induced by $x$, begun from position $c$ at time $s$. Our main result reads:

Theorem 1. Let $\mathfrak{p}_{\lambda}=\mathfrak{p}_{\lambda, \beta, a}$ denote the right hand side of (3). For large values of $\lambda$ it holds

$$
\mathfrak{p}_{\lambda}=e^{-\frac{\beta}{2} \lambda+2 \gamma \sqrt{\lambda}} \lambda^{-\frac{\gamma(\gamma+1-\beta / 2)}{2 \beta}} \mathfrak{e}(\beta, a)(1+o(1)) .
$$

Here $\gamma=\frac{\beta}{2}(a+1)-1$ and $\mathfrak{e}(\beta, a)>0$ is an undetermined constant.

There has already been a great deal of work in this direction, though focussed on dealing directly with the statistics (1) rather than our passage time description (3). The fundamental treatment of Tracy-Widom [13] for $\beta=2$ produced the correct $\lambda \rightarrow \infty$ asymptotics of $\mathfrak{p}_{\lambda, 2, a}$ up to a multiplicative constant and provided a conjecture for that constant, $\mathfrak{e}(2, a)$. This has recently been verified by Ehrhardt [6], for $|a|<1$, by operator theoretic techniques, and for all $a>-1$ by DeiftKrasovsky-Vasilevska [2] using Riemann Hilbert Problem machinery. A non-rigorous argument in [1] predicted all factors in the asymptotics save the constant for all $(\beta, a)$. Making use of integral identities available at special values of $\beta$ and integer $a$, Forrester has a sound conjecture for the value of the general constant $\mathfrak{e}(\beta, a)$, see [7]. It appears possible that a type of analytic continuation argument could extend the result of [7] to all $\beta$ (though for still integer $a$ ). The method employed here leaves $\mathfrak{e}(\beta, a)$ in opaque form, as a somewhat involved expectation over diffusion paths; an explicit determination of this object for all $\beta$ and $a$ remains an open problem.

In many ways, the chief insight of this paper is to cast the diffusion (2), which encodes the desired probability distribution, in the present form. (The process which appears in [10] is related by a change of variables.) In fact, $t \mapsto x(t)$ is remarkably similar to the process studied by Valkó-Virág 
in estimating the probability of large gaps in the general beta "bulk" [14]. They showed that the probability of a gap being larger than $\lambda$ is equal to the non-explosion, again to $-\infty$, of

$$
d z(t)=d b(t)+\left(\frac{1}{2} \tanh z(t)-\frac{\beta}{8} \lambda e^{-\beta t / 4} \cosh z(t)\right) d t,
$$

begun again at $+\infty$. It is no surprise then that their basic argument, which involves estimating the Cameron-Martin-Girsanov factor produced by a well-chosen change of measure, may be followed in this case.

The proof of Theorem 1 occupies sections 3 and 4; section 2 gives a self-contained explanation of the identity (3).

\section{Passage time description for $\Lambda$}

Without pointing the reader to [10] and the subsequent erratum, it is easy enough to give a brief derivation of the relevance of the diffusion (2) to the distribution function $P(\Lambda>\lambda)$. The main result of [10] shows that $\Lambda^{-1}$ is the maximal eigenvalue of the almost surely trace class integral operator

$$
L_{\beta, a} \psi(t):=\int_{0}^{\infty}\left(\int_{0}^{t \wedge s} e^{a u+\frac{2}{\sqrt{\beta}} b(u)} d u\right) \psi(s) e^{-(a+1) s-\frac{2}{\sqrt{\beta}} b(s)} d s,
$$

acting on $L^{2}\left[\mathbb{R}_{+}, \mu\right], \mu(d t)=e^{-(a+1) t-\frac{2}{\sqrt{\beta}} b(t)} d t$. Here $t \mapsto b(t)$ is a standard Brownian motion.

Any nonnegative $L^{2}$ solution of $\psi(t)=\lambda L_{\beta, a} \psi(t)$ satisfies $\psi(0)=0$ and $\psi^{\prime}(t):=d \psi(t) / d t \geq 0$ for all $t>0$, as can be seen by taking derivatives of both sides of the eigenvalue equation:

$$
\psi^{\prime}(t)=\lambda e^{a t+\frac{2}{\sqrt{\beta}} b(t)} \int_{t}^{\infty} \psi(s) e^{-(a+1) s-\frac{2}{\sqrt{\beta}} b(s)} d s .
$$

This converts to a differential system:

$$
d \psi^{\prime}(t)=\frac{2}{\sqrt{\beta}} \psi^{\prime}(t) d b(t)+\left[\left(a+\frac{2}{\beta}\right) \psi^{\prime}(t)-\lambda e^{-t} \psi(t)\right] d t, d \psi(t)=\psi^{\prime}(t) d t
$$

which can be used to test whether a fixed $\lambda$ is at or below an eigenvalue. Specifically, $\lambda$ is strictly below the groundstate eigenvalue $\Lambda$ if the solution to (6) begun at $\psi(0)=0$ (and $\psi^{\prime}(0)=1$ say) satisfies $\psi(t)>0, \psi^{\prime}(t)>0$ for all time (note that solutions of (6) are decreasing in $\lambda$ ). It is now the standard trick to translate this condition onto the diffusion $q(t):=\psi^{\prime}(t) / \psi(t)$ which solves

$$
d q(t)=\frac{2}{\sqrt{\beta}} q(t) d b(t)+\left[\left(a+\frac{2}{\beta}\right) q(t)-q^{2}(t)-\lambda e^{-t}\right] d t,
$$

started from $+\infty$ at time $t=0$. In particular, if $\tau_{c}$ is the passage time of $q$ to a level $c$, the event $\{\Lambda>\lambda\}$ coincides with $\left\{\tau_{0}=\infty\right\}$. Now the change of variables,

$$
x(t):=\log (q(\beta t / 4))+\beta t / 8-\log \lambda / 2,
$$

explains the identity (3). 
As a bit of amplification, we remark that for $q=q(\cdot ; a, \beta, \lambda)$ with $a \geq 0$,

$$
\mathbb{P}\left(\tau_{-\infty}(q)<\infty \mid \tau_{0}(q)<\infty\right)=1
$$

So, at least for $a \geq 0$, one can replace the condition of $q$ never vanishing with the (more familiar) condition that $q$ never explodes to $-\infty$. Furthermore, a change of variables similar to (7) shows that the event that $q(\cdot ; a, \beta, \lambda)$ started from 0 never hits $-\infty$ is the same as the event that $q(\cdot ;-a-$ $1, \beta, \lambda)$, started from $+\infty$ never hits 0 . In particular, defining instead $x(t):=-\log (-q(\beta t / 4))+$ $\beta t / 8-\log \lambda / 2$ (keeping in mid that for all $t>\tau_{0}, q(t)<0$ ), $x(t)$ will solve (2) with $a$ replaced by $-a-1$. One concludes that

$$
\lim _{a \downarrow-1} P(\Lambda>\lambda)=\lim _{a \downarrow-1} \mathbb{P}_{\infty}\left(\tau_{0}(q(\cdot ; a, \beta, \lambda))=\infty\right)=\lim _{a \uparrow 0} \mathbb{P}_{0}\left(\tau_{-\infty}(q(\cdot ; a, \beta, \lambda))=\infty\right)=0
$$

for any $\lambda>0$ (by say monotone convergence), as would have been guessed ahead of time.

To prove (8), on the event $\left\{\tau_{0}<\infty\right\}$ introduce the simpler change of variables $u(t)=\log (-q(t+$ $\left.\left.\tau_{0}\right)\right)$. This process satisfies

$$
d u(t)=\frac{2}{\sqrt{\beta}} d b(t)+\left[a+e^{u(t)}+\lambda e^{-\tau_{0}} e^{-t} e^{-u(t)}\right] d t, u(0+) \in(-\infty, \infty),
$$

to which we compare the homogeneous process defined by

$$
d v(t)=\frac{2}{\sqrt{\beta}} d b(t)+\left[a+e^{v(t)}\right] d t, \quad v(0)=u(0+) \in(-\infty, \infty) .
$$

As $u(t)>v(t), q$ explodes to $-\infty$ in finite time if $v$ explodes to $+\infty$ in finite time (we continue to work on the event $\left.\left\{\tau_{0}(q)<\infty\right\}\right)$.

Now apply Feller's test, in the form given by Proposition 5.32 (part (ii)) of [9]. In particular, bring in the Lyapunov function

$$
m(x)=\int_{0}^{x} s(y) \int_{0}^{y} \frac{1}{s(z)} d z d y \text { where } s(x)=\exp \left(-\frac{\beta}{2} \int_{0}^{x}\left(a+e^{z}\right) d z\right)
$$

$(s(x)$ is the derivative of the scale function for $v)$. Since

$$
\lim _{x \rightarrow \infty} m(x)<\infty, \text { while, if } a \geq 0, \lim _{x \rightarrow-\infty} m(x)=-\infty,
$$

the cited form of Feller's test implies that $S=\int\{t: v(t) \notin(-\infty, \infty)\}$ is finite with probability one. However, it is impossible that $v(t)$ ever hits $-\infty$ (it is easily bounded below by a Brownian motion with constant drift $a$ ). This completes the proof.

\section{Change of measure}

Hereafter it is convenient to put the time index in subscripts, i.e., $x(t)$ becomes $x_{t}$ and the like. To begin, introduce the notation

$$
\mathfrak{p}_{\lambda}(c)=\mathbb{P}_{c}\left(x_{t} \text { never explodes }\right)
$$


Then, by the strong Markov property,

$$
\mathfrak{p}_{\lambda}=\mathfrak{p}_{\lambda}(\infty)=\mathbb{E}_{\infty}\left[\mathfrak{p}_{1}\left(x_{T}\right), x_{t}>-\infty \text { for } t \in(0, T]\right]
$$

upon choosing

$$
T=\frac{4}{\beta} \log \lambda
$$

The change of measure is now enacted on the expectation (9).

Proposition 2. Let $h(t, x)$ be $C^{1}$ in both variables and bounded for $t \leq T$. Then, the law on paths up to time $T$ induced by

$$
d y_{t}=d b_{t}+\left(h\left(t, y_{t}\right)-\frac{\beta}{2} \sqrt{\lambda} e^{-\beta t / 8} \sinh \left(y_{t}\right)\right) d t, y_{0}=\infty
$$

is absolutely continuous with respect to that of $t \mapsto x_{t}, x_{0}=\infty$, subject to $x_{t}>-\infty, 0 \leq t \leq T$. Moreover,

$$
\mathfrak{p}_{\lambda}=\mathbb{E}_{\infty}\left[\mathfrak{p}_{1}\left(y_{T}\right) R_{T}(y .)\right]
$$

in which, for $s \leq T$,

$$
\log R_{s}(y)=\int_{0}^{s}\left(f\left(t, y_{t}\right)-g\left(t, y_{t}\right)\right) d y_{t}-\frac{1}{2} \int_{0}^{s}\left(f^{2}\left(t, y_{t}\right)-g^{2}\left(t, y_{t}\right)\right) d t,
$$

$f(t, y)=\frac{\beta}{4}\left(a+\frac{1}{2}\right)-\frac{\beta}{2} \sqrt{\lambda} e^{-\beta t / 8} \cosh y$ and $g(t, y)=h(t, y)-\frac{\beta}{2} \sqrt{\lambda} e^{-\beta t / 8} \sinh y$.

This is just the formula of Cameron-Martin-Girsanov, applied to the particular case of a diffusion with explosion for which it is important to point out that the test function $\mathfrak{p}_{1}\left(x_{T}\right)$ in question vanishes when $T$ is larger than the explosion time. One also notes that the general form of the $y$-drift, effectively a bounded function minus $\sinh y$, allows $y_{t}$ to be started at $+\infty$ and prevents $y_{t}$ from exploding on $[0, T]$. To then carry out the standard proof of Cameron-Martin-Girsanov in the present context, it must be checked that $R_{t}^{-1}(x)$, which is a local martingale by construction, is actually a martingale. But again by the general form of the $y$-drift, both $f-g$ and $f^{2}-g^{2}$ are bounded when the path is bounded below, keeping $R_{t}^{-1}(x)$ bounded prior to the explosion time of $x_{t}$. Plainly, $R_{t}^{-1}(x)=0$ at and after the explosion time.

The first ingredient of the proof of Theorem 1 is the following. Throughout the below, $[x]^{-}$ denotes the negative part of $x \in \mathbb{R}$. Also recall that $\gamma=\frac{\beta}{2}(a+1)-1$.

Lemma 3. There exists a choice of $h$ in Proposition 2 so that, for appropriate $v$, $\phi$ satisfying $|v(t, y)| \leq$ $\kappa_{1}+\kappa_{2}[y]^{-}$for all $t \geq 0$ and constant $\kappa_{1}, \kappa_{2}$, and $|\phi(t, y)| \leq \hat{\phi}(t)$ with $\int_{0}^{\infty} \hat{\phi}(t) d t<\infty$, it holds that

$$
\begin{aligned}
\log R_{T}\left(y_{.}\right) & =-\frac{\beta}{2} \lambda+2 \gamma \sqrt{\lambda}-\left(\frac{\gamma(\gamma+1-\beta / 2)}{2 \beta}\right) \log \lambda \\
& +\frac{\beta}{2} e^{-y_{T}}+v\left(T, y_{T}\right)+\int_{0}^{T} \phi\left(T-t, y_{t}\right) d t
\end{aligned}
$$


Once $h$ is in hand, the lemma is readily verified. In particular,

$$
h(t, y)=\frac{\beta}{4}\left(a+\frac{1}{2}\right)+h_{1}(y)+e^{-\frac{\beta}{8}(T-t)} h_{2}(y)
$$

where

$$
\begin{aligned}
& h_{1}(y)=-\frac{\gamma}{1+e^{y}}, \\
& h_{2}(y)=\frac{1}{\beta \sinh (y)}\left(\left(h_{1}^{2}(y)-h_{1}^{2}(0)\right)+\frac{\beta}{2}\left(a+\frac{1}{2}\right)\left(h_{1}(y)-h_{1}(0)\right)+\left(h_{1}^{\prime}(y)-h_{1}^{\prime}(0)\right)\right) .
\end{aligned}
$$

That both $h_{1}$ and $h_{2}$ are uniformly bounded, $h_{1}$ being integrable at $+\infty$ while $h_{2}$ is integrable at both $\pm \infty$ figure into the bounds on $v$ and $\phi$ in the lemma.

It is more instructive however to describe how $h$ is discovered, each step achieving successive order in $\lambda, \sqrt{\lambda}$, etc., and the various bounds claimed in the lemma seen along the way.

Step 1 begins by expanding out the exponential $R_{T}$ factor with a generic $h$ :

$$
\begin{aligned}
\log R_{T}(y .) & =-\frac{\beta^{2}}{8} \lambda \int_{0}^{T} e^{-\beta t / 4} d t+\frac{1}{2} \int_{0}^{T} h^{2}\left(t, y_{t}\right) d t-\frac{\beta^{2}}{32}\left(a+\frac{1}{2}\right)^{2} T \\
& -\frac{\beta}{2} \sqrt{\lambda} \int_{0}^{T} e^{-\beta t / 8} h\left(t, y_{t}\right) \sinh \left(y_{t}\right) d t+\frac{\beta^{2}}{8}\left(a+\frac{1}{2}\right) \sqrt{\lambda} \int_{0}^{T} e^{-\beta t / 8} \cosh \left(y_{t}\right) d t \\
& -\frac{\beta}{2} \sqrt{\lambda} \int_{0}^{T} e^{-\beta t / 8} e^{-y_{t}} d y_{t}-\int_{0}^{T}\left[h\left(t, y_{t}\right)-\frac{\beta}{4}\left(a+\frac{1}{2}\right)\right] d y_{t} .
\end{aligned}
$$

By the choice of $T$, the first term equals $-\frac{\beta}{2}(\lambda-1)$ which already gives the leading order and explains the particulars of the sinh $y$ term in the choice of the $y$-drift. The last term, coupled with the fact that $y_{0}=\infty$, prompts a natural shift of $h$ by the factor $\frac{\beta}{4}\left(a+\frac{1}{2}\right)$. That is, $h$ is replaced with $h+\frac{\beta}{4}\left(a+\frac{1}{2}\right)$.

Step 2 enacts the above shift, and also introduces the obvious Itô substitution in the second last term of (15),

$$
\frac{\beta}{2} \sqrt{\lambda} \int_{0}^{T} e^{-\beta t / 8} e^{-y_{t}} d y_{t}=-\frac{\beta}{2} e^{-y_{T}}+\left(\frac{\beta}{4}-\frac{\beta^{2}}{16}\right) \sqrt{\lambda} \int_{0}^{T} e^{-\beta t / 8} e^{-y_{t}} d t
$$

to write:

$$
\begin{aligned}
\log R_{T}(y .) & =-\frac{\beta^{2}}{8} \lambda \int_{0}^{T} e^{-\beta t / 4} d t \\
& +\frac{\beta}{2} \sqrt{\lambda}\left(\frac{\gamma}{2} \int_{0}^{T} e^{-\beta t / 8} e^{-y_{t}} d t-\int_{0}^{T} e^{-\beta t / 8} h\left(t, y_{t}\right) \sinh \left(y_{t}\right) d t\right) \\
& +\frac{1}{2} \int_{0}^{T} h^{2}\left(t, y_{t}\right) d t+\frac{\beta}{4}\left(a+\frac{1}{2}\right) \int_{0}^{T} h\left(t, y_{t}\right) d t-\int_{0}^{T} h\left(t, y_{t}\right) d y_{t}+\frac{\beta}{2} e^{-y_{T}} .
\end{aligned}
$$


This draws attention to line two of (16), which should produce the final constant times $\sqrt{\lambda}$ term. This may be achieved most easily by introducing a deterministic integrand in that line via the substitution

$$
h(t, y)=\frac{\gamma}{2} \frac{e^{-y}-1}{\sinh (y)}+\bar{h}(t, y):=h_{1}(y)+\bar{h}(t, y)
$$

so that

$$
\begin{aligned}
\frac{\gamma}{2} \int_{0}^{T} e^{-\beta t / 8} e^{-y_{t}} d t-\int_{0}^{T} e^{-\beta t / 8} h\left(t, y_{t}\right) \sinh \left(y_{t}\right) d t \\
=\frac{\gamma}{2} \int_{0}^{T} e^{-\beta t / 8} d t-\int_{0}^{T} e^{-\beta t / 8} \bar{h}\left(t, y_{t}\right) \sinh \left(y_{t}\right) d t
\end{aligned}
$$

Evaluating all deterministic factors thus far, step 2 is summarized by

$$
\begin{aligned}
\log R_{T}(y) & =-\frac{\beta}{2} \lambda+2 \gamma \sqrt{\lambda}+\frac{\beta}{2} e^{-y_{T}}-\left(\beta\left(a+\frac{1}{2}\right)+2\right) \\
& -\frac{\beta}{2} \sqrt{\lambda} \int_{0}^{T} e^{-\beta t / 8} \bar{h}\left(t, y_{t}\right) \sinh \left(y_{t}\right) d t \\
& +\frac{1}{2} \int_{0}^{T} h^{2}\left(t, y_{t}\right) d t+\frac{\beta}{4}\left(a+\frac{1}{2}\right) \int_{0}^{T} h\left(t, y_{t}\right) d t-\int_{0}^{T} h\left(t, y_{t}\right) d y_{t} .
\end{aligned}
$$

The first two terms above exhibit the proposed order $\lambda$ and order $\sqrt{\lambda}$ factors in the statement of the lemma, showing that there was not much flexibility in the choice of the (uniformly bounded) function $h_{1}$ in (17).

Step 3 is to pin down the $\log \lambda$ factor in the exponent (or, equivalently, the $T$ factor). A look at line two of (18) suggests a prescription for $\bar{h}$ :

$$
\bar{h}(t, y)=\frac{2}{\beta \sqrt{\lambda}} e^{\beta t / 8} h_{2}(y)=\frac{2}{\beta} e^{-(\beta / 8)(T-t)} \frac{h_{3}(y)}{\sinh (y)},
$$

in which $h_{3}$ must be chosen so that $h_{2}$ is bounded (and more).

With $\eta(t)=\frac{2}{\beta} e^{-\beta t / 8}$, we employ Itô's lemma once more to write the final term in (18) as in

$$
\begin{aligned}
\int_{0}^{T} h\left(t, y_{t}\right) d y_{t} & =H_{1}\left(y_{T}\right)+H_{2}\left(T, y_{T}\right) \\
& -\frac{1}{2} \int_{0}^{T} h_{1}^{\prime}\left(y_{t}\right) d t+\int_{0}^{T} \eta^{\prime}(T-t)\left[\int_{0}^{y_{t}} h_{2}(z) d z\right] d t-\frac{1}{2} \int_{0}^{T} \eta(T-t) h_{2}^{\prime}\left(y_{t}\right) d t .
\end{aligned}
$$

Here $H_{1}$ and $H_{2}$ denote the anti-derivative terms which appear: $H_{1}\left(y_{T}\right)=\left.\int_{0}^{y_{t}} h_{1}(y) d y\right|_{0} ^{T}$ and $H_{2}\left(T, y_{T}\right)=\left.\eta(T-t) \int_{0}^{y_{t}} h_{2}(y) d y\right|_{0} ^{T}$. Note that the boundary values of $H_{2}$ will necessitate that our choice of $h_{2}$, like that of $h_{1}$, is integrable at $+\infty\left(=y_{0}\right)$. Now expand out the last two lines of 
(18) to read:

$$
\begin{aligned}
& \int_{0}^{T}\left[\frac{1}{2} h_{1}^{2}\left(y_{t}\right)+\frac{1}{2} h_{1}^{\prime}\left(y_{t}\right)+\frac{\beta}{4}\left(a+\frac{1}{2}\right) h_{1}\left(y_{t}\right)-h_{3}\left(y_{t}\right)\right] d t+H_{1}\left(y_{T}\right)+H_{2}\left(T, y_{T}\right) \\
& +\int_{0}^{T}\left[\frac{1}{2} \eta^{2}(T-t) h_{2}^{2}\left(y_{t}\right)\right. \\
& \left.\quad+\eta(T-t)\left(h_{1}\left(y_{t}\right) h_{2}\left(y_{t}\right)+\frac{\beta}{4}\left(a+\frac{1}{2}\right) h_{2}\left(y_{t}\right)+\frac{\beta}{8}\left[\int_{0}^{y_{t}} h_{2}(y) d y\right]+\frac{1}{2} h_{2}^{\prime}\left(y_{t}\right)\right)\right] d t .
\end{aligned}
$$

The first term of (21) prompts the choice of $h_{3}$, namely set

$$
h_{3}(y)=\frac{1}{2} h_{1}^{2}(y)+\frac{1}{2} h_{1}^{\prime}(y)+\frac{\beta}{4}\left(a+\frac{1}{2}\right) h_{1}(y)-\kappa,
$$

for a constant $\kappa$ which makes $h_{2}(y)=h_{3}(y) / \sinh (y)$ bounded. We find that

$$
\kappa=\frac{1}{2} h_{1}^{2}(0)+\frac{1}{2} h_{1}^{\prime}(0)+\frac{\beta}{4}\left(a+\frac{1}{2}\right) h_{1}(0)=\frac{\gamma(\gamma+1)}{8}-\frac{\beta}{8}\left(a+\frac{1}{2}\right) \gamma=-\frac{\gamma}{8}(\gamma+1-\beta / 2),
$$

compare (14). In other words, with this choice the first term of (21) equals $\frac{4}{\beta} \kappa \log \lambda$, the advertised $\log \lambda$ contribution in Theorem 1.

To finish the proof of the lemma we further identify

$$
v(T, y)=-\left(\beta\left(a+\frac{1}{2}\right)+2\right)+H_{1}(y)+H_{2}(T, y),
$$

and $\phi(T-t, y)$ with the integrand of the last term in (21). One now checks: $h_{1}$ and $h_{2}$ along with their derivatives are uniformly bounded over the entire real line (with constants depending on $a$ and $\beta$ of course), $h_{1}$ is integrable at $+\infty$, and $h_{2}$ is in fact integrable at both $\pm \infty$. The boundedness and integrability (at $+\infty$ ) of $h_{1}$ and $h_{2}$ give immediately that $\left|H_{1}(y)\right|+\left|H_{2}(t, y)\right| \leq$ $\kappa_{1}+\kappa_{2} y^{-}$, proving the claimed bound on $v$. For $\phi$ one shows that $|\phi(T-t, y)| \leq c \eta(T-t)$, using the additional appraisals on the derivatives, that $\int_{-\infty}^{\infty}\left|h_{2}\right|<\infty$, and the simple fact $0<\eta(T-t) \leq$ $\eta(0)$ for $t \in[0, T]$.

\section{Constant term}

The conclusion of the previous section is that

$$
\mathfrak{p}_{\lambda}=e^{-\frac{\beta}{2} \lambda+2 \gamma \sqrt{\lambda}} \lambda^{\left.-\frac{\gamma(\gamma+1-\beta / 2)}{2 \beta}\right) \gamma} \mathfrak{e}_{\lambda}
$$

with

$$
\mathfrak{e}_{\lambda}=\mathbb{E}_{\infty}\left[\mathfrak{p}_{1}\left(y_{T}\right) e^{\frac{\beta}{2} e^{-y_{T}}+v\left(y_{T}\right)+\int_{0}^{T} \phi\left(T-t, y_{t}\right) d t}\right],
$$

and $v$ and $\phi$ satisfying the bounds outlined in Lemma 3. It remains to show that the existence of a (non-zero) constant $\mathfrak{e}=\mathfrak{e}(a, \beta)$ such that $\lim _{\lambda \rightarrow \infty} \mathfrak{e}_{\lambda}=\mathfrak{e}$. This is again structurally identical to [14]. 
The first observation is that the $\mathbb{E}_{\infty}$ integration is performed over paths that are monotonically decreasing in $T$. The nicest way to see this is to replace the integration over $y_{t}, 0 \leq t \leq T$ with that over

$$
y_{t}^{T}=y_{t+T}, \quad-T \leq t \leq 0
$$

which satisfies

$$
d y_{t}^{T}=d b_{t}+\left(h\left(t+T, y_{t}^{T}\right)-\frac{\beta}{2} e^{-t} \sinh y_{t}^{T}\right) d t, \quad y_{-T}^{T}=\infty .
$$

If this family of processes is run on the same Brownian motion, $t \mapsto b_{t}$, it follows that $y_{t}^{T_{1}} \leq y_{t}^{T_{2}}$ for $t \geq-T_{2}$ : by definition $y_{-T_{2}}^{T_{1}}<y_{-T_{2}}^{T_{2}}$ and the evolution maintains the ordering. Denote this sequence of corresponding expectations simply by $\mathbf{E}$ and record that

$$
\mathfrak{e}_{\lambda}=\mathbf{E}\left[\mathfrak{p}_{1}\left(y_{0}^{T}\right) e^{\psi\left(y^{T}\right)}\right], \quad \psi\left(y^{T}\right)=\frac{\beta}{2} e^{-y_{0}^{T}}+v\left(T, y_{0}^{T}\right)+\int_{0}^{T} \phi\left(t, y_{-t}^{T}\right) d t .
$$

Next, pick a constant $h_{0}$ such that

$$
\inf _{-\infty<y<\infty,-T<t<0} h(t+T, y)>h_{0}
$$

(a look at (13) and (14) shows this is possible), and introduce the stationary diffusion $t \mapsto z_{t}$ on the negative half-line with generator

$$
\mathscr{L}=\frac{1}{2} \frac{d^{2}}{d z^{2}}+f(z) \frac{d}{d z}, \quad f(z)=h_{0}-\frac{\beta}{2} \sinh z,
$$

and reflected (downward) at the origin. In particular, for all $t \geq-T, \mathbb{P}\left(z_{t} \in d z\right)=\mathfrak{m}(d z)$ where

$$
\mathfrak{m}(d z)=\kappa_{0} e^{2 h_{0} z-\beta \cosh z} d z, \quad z \in(-\infty, 0],
$$

and $\kappa_{0}$ is the appropriate normalizer. This is the well-known formula for the speed measure (see for example [8]), or one may check that $\int_{-\infty}^{0} \mathscr{L} \phi(z) \mathfrak{m}(d z)=0$ for all smooth $\phi$ satisfying $\phi^{\prime}(0)=0$.

Again running $z_{t}$ on the same Brownian motion, it holds that $y_{t}^{T} \geq z_{t}>-\infty$ for all $t \in[-T, 0]$. This is plain at the starting time, and continues by the domination (from below) of the $y^{T}$-drift by that of $z$. It follows that there exists a random variable $y_{t}^{\infty}>-\infty$ such that

$$
\lim _{T \rightarrow \infty} y_{t}^{T}=y_{t}^{\infty} \text { almost surely for each } t \in(-\infty, 0] \text {. }
$$

To pass this convergence under the E-expectation we prepare the following (and defer the proof to the end of the section).

Lemma 4. The function $x \mapsto \mathfrak{p}_{1}(x)$ is continuous, strictly positive on $x>-\infty$ and satisfies

$$
\mathfrak{p}_{1}(x) \leq \kappa_{3} e^{-\frac{\beta}{4} e^{-x}}
$$

for a constant $\kappa_{3}$. 
Courtesy (26) and the first statement in Lemma 4 we have that

$$
\begin{aligned}
& \lim _{T \rightarrow \infty} \mathfrak{p}_{1}\left(y_{0}^{T}\right) e^{\psi\left(y^{T}\right)}=\mathfrak{p}_{1}\left(y_{0}^{\infty}\right) e^{\frac{\beta}{2} e^{-y_{0}^{\infty}}+v\left(\infty, y_{0}^{\infty}\right)+\int_{0}^{\infty} \phi\left(t, y_{-t}^{\infty}\right) d t} \\
& :=\mathfrak{p}_{1}\left(y_{0}^{\infty}\right) e^{\psi_{\infty}\left(y^{\infty}\right)},
\end{aligned}
$$

using continuity (for the first three factors) and dominated convergence (for the last factor). The evaluation $\left.v(t, y)\right|_{t=\infty}$ simply has the effect of setting of one $H_{2}$-terms of which $v$ is comprised to zero, recall (22).

Next, by the estimates on $v, \phi$ from Lemma 3 and (27), there are the bounds

$$
\kappa_{4}^{-1} \mathfrak{p}_{1}\left(y_{0}^{T}\right) e^{-\kappa_{5}\left[y_{0}^{T}\right]^{-}} \leq \mathfrak{p}_{1}\left(y_{0}^{T}\right) e^{\psi\left(y^{T}\right)} \leq \kappa_{4} e^{\kappa_{5}\left[y_{0}^{T}\right]^{-}+\left(\frac{\beta}{2}-\frac{\beta}{4}\right) e^{-\left[y_{0}^{T}\right]^{-}}},
$$

with positive constants $\kappa_{4}, \kappa_{5}$.

Note that both bounds in (29) depend only on the marginal of the process at time 0 , and denote the left and right hand sides by $\mathfrak{p}_{-}\left(y_{0}^{T}\right)$ and $\mathfrak{p}_{+}\left(y_{0}^{T}\right)$ respectively. Invoking again the path-wise control, $y_{t}^{T} \geq z_{t}, t \in[-T, 0]$ we have that

$$
\mathfrak{p}_{1}\left(y_{0}^{T}\right) e^{\psi\left(y^{T}\right)} \leq \mathfrak{p}_{+}\left(z_{0}\right), \quad \mathbf{E}\left[\mathfrak{p}_{+}\left(z_{0}\right)\right]=\int_{-\infty}^{0} \mathfrak{p}_{+}(z) \mathfrak{m}(d z)<\infty,
$$

where $\mathfrak{m}$ is defined in (25). Returning to (23), (28) and dominated convergence now produce

$$
\lim _{\lambda \rightarrow \infty} \mathfrak{e}_{\lambda}=\lim _{T \rightarrow \infty} \mathbf{E}\left[\mathfrak{p}_{1}\left(y_{0}^{T}\right) e^{\psi\left(y^{T}\right)}\right]=\mathbf{E}\left[\mathfrak{p}_{1}\left(y_{0}^{\infty}\right) e^{\psi_{\infty}\left(y^{\infty}\right)}\right]:=\mathfrak{e},
$$

defining the constant $\mathfrak{e}$ in the statement of Theorem 1 . That $\mathfrak{e}$ is not equal to zero follows from

$$
\mathfrak{e} \geq \liminf _{T \rightarrow \infty} \mathbf{E}\left[\mathfrak{p}_{1}\left(y_{0}^{T}\right) e^{\psi\left(y^{T}\right)}\right] \geq \int_{-\infty}^{0} \mathfrak{p}_{-}(z) \mathfrak{m}(d z)>0 .
$$

Here we have used that $z \mapsto \mathfrak{p}_{-}(z)$ is decreasing in order to replace $y^{T}$-paths with $z$-paths, along with the fact that $\mathfrak{p}_{1}(z)$ (and so too $\mathfrak{p}_{-}(z)$ ) is strictly positive (Lemma 4 ). This completes the proof of Theorem 1, granted the below.

Proof of Lemma 4. The continuity follows from that of the transition density $p(\cdot, x, y)$ in both space variables (the corresponding generator is hypo-elliptic).

To see that $\mathfrak{p}_{1}(x)>0$, first note that the operator $L_{\beta, a}$ defined in (5) which encodes the point process of eigenvalues is positive and compact. A proof that $L_{\beta, a}$ is in fact (almost surely) trace class is contained in Lemma 6 of [10]. Its maximal eigenvalue, $\Lambda^{-1}$, is therefore almost surely bounded above, and so there exists a small enough $\lambda_{0}>0$ such that $\mathfrak{p}_{\lambda_{0}}=\mathfrak{p}_{\lambda_{0}}(\infty)>0$. Next, by the Markov property,

$$
\mathfrak{p}_{\lambda_{0}}(\infty)=\int_{\infty}^{\infty} p(t, \infty, x) \mathfrak{p}_{\lambda_{0} e^{-\beta t / 4}}(x) d x
$$

and it follows that for every $t>0$ there is a $x_{0}$ such that $\mathfrak{p}_{\lambda_{0} e^{-\beta t / 4}}\left(x_{0}\right)>0$. Applying the same formula once again, we find that for any $z \in \mathbb{R}$

$$
\mathfrak{p}_{1}(x) \geq \int_{z}^{\infty} p(s, x, y) \mathfrak{p}_{e^{-\beta s / 4}}(y) d y \geq \mathbb{P}_{x}\left(x_{s} \geq z\right) \mathfrak{p}_{e^{-\beta s / 4}}(z) .
$$


To finish, choose $s=t-\frac{4}{\beta} \log \lambda_{0}$ and then set $z$ to be the appropriate $x_{0}$.

For the bound (27) we can restrict to $x$ less than some large negative constant, and note that $\mathfrak{p}_{1}(x)$ is bounded by the probability of non explosion for the following process

$$
d \tilde{y}_{t}=d b_{t}+\frac{\beta}{4}\left(a+\frac{1}{2}\right)-\frac{\beta}{4} e^{-\beta t / 8} e^{-\tilde{y}_{t}} .
$$

since the downward drift on $\tilde{y}$ is weaker than that of $x$. Next make the change $y_{t}=\tilde{y}_{t}+\frac{\beta t}{8}$ to obtain the homogenous process

$$
d y_{t}=d b_{t}+\frac{\beta}{4}\left(a+1-e^{-y_{t}}\right),
$$

to which we can apply a version of Feller's test, similar to what was done at the end of Section 2. A scale function for the $y$-process is

$$
s(y)=\int_{0}^{y} \exp \left\{-\frac{\beta}{2}\left[(a+1) \xi+e^{-\xi}-1\right]\right\} d \xi,
$$

and the probability that this process exits through $+\infty$ is exactly the probability of not exploding. This follows by checking the conditions of now Proposition 5.22 of [9]. According to that same proposition, the exit probability equals

$$
\frac{s(x)-s(-\infty)}{s(+\infty)-s(-\infty)}=\frac{1}{Z} \int_{-\infty}^{x} \exp \left\{-\frac{\beta}{2}\left[(a+1) \xi+e^{-\xi}\right]\right\} d \xi
$$

from which the required bound easily follows.

Acknowledgements We thank the anonymous referee for a close reading of the submitted version.

\section{References}

[1] Chen, Y., Manning, S.M. (1994) Asymptotic level spacing of the Laguerre ensemble: a coulomb fluid approach. J. Phys. A: Math. Gen. 27, 3615-3620. MR1282574

[2] Deift, P., Krasovsky, I., Vasilevska, J. (2011) Asymptotics for a determinant with a confluent hypergeometric kernel. Int. Math. Res. Not. 2011, 2117-2160. MR2806560

[3] Dumitriu, I., Edelman, A. (2002) Matrix models for beta ensembles. J. Math. Phys. 43, 5830-5847. MR1936554

[4] Edelman, A. (1988) Eigenvalues and condition numbers of random matrices. SIAM J. Matrix Anal. Appl. 9, 543-560. MR0964668

[5] Edelman, A., Sutton, B. (2007) From random matrices to stochastic operators. J. Stat. Phys. 127, 1121-1165. MR2331033

[6] Ehrhardt, T. (2010) The asymptotics of a Bessel-kernel determinant which arises in Random Matrix Theory. Adv. Math. 225, 3088-3133. MR2729003 
[7] ForRester, P.J. (1994) Exact results and universal asymptotics in the Laguerre random matrix ensemble. J. Math. Phys. 35, no. 5, 2539-2551. MR1271945

[8] Itô, K., McKean, H.P. Diffusion processes and their sample paths. Springer-Verlag, BerlinHeidelberg-New York, 1974. MR0345224

[9] Karatzas, I., Shreve, S.E. Brownian Motion and Stochastic Calculus. Second edition, Springer, New York, 1991. MR1121940

[10] Ramírez, J., Rider, B. (2009) Diffusion at the random matrix hard edge. Comm. Math. Phys. 288, 887-906. (Erratum CMP 307 (2011), 561-563.) MR2504858

[11] Rudelson, M., Vershynin, R. (2010) Non-asymptotic theory of random matrices: extreme singular values. Proceedings of the International Congress of Mathematicians. Hyderabad, India.

[12] TAO, T., VU, V. (2010) Random matrices: The distribution of the smallest singular values. GAFA 20, no. 1, 260-297. MR2647142

[13] Tracy, C., Widom, H. (1994) Level spacing distributions and the Bessel kernel. Comm. Math. Phys. 161 no. 2, 289-309. MR1266485

[14] Valkó, B., ViráG, B. (2010) Large gaps between random eigenvalues. Ann. Probab. 38, no. 3, 1263-1279. MR2674999 